\title{
Impact Dynamic Analysis of Multibody System Based on Impulse Momentum Method
}

\author{
Yuechen Duan, Tingting Wang and ${\mathrm{Xia} \mathrm{Li}^{*}}^{*}$ \\ School of Mechanical Engineering, Zhengzhou University, No. 100 of Science Road, Zhengzhou, China 450001 \\ ${ }^{*}$ Corresponding author
}

\begin{abstract}
The impact dynamics of flexible multibody systems is analyzed based on the impulse momentum method (IMM). According to the rigid-flexible coupling dynamic theory of flexible multibody system, the continuous dynamic equations of the system are established by the recursive Lagrange method. By using IMM, the impact dynamic equations of the system are obtained. The impact process is assumed to be timeless, and the generalized velocity mutates at the impact moment. The contact criterion is given. An impact dynamic simulation example for a two-link flexible multibody system is given, as well as the global dynamic behaviors. The results show that the impact dynamic solving method based on IMM can be used for the global impact dynamics of flexible multibody systems. Impact affects the dynamic behaviors of the system during and after impact. The large overall motion, the small deformation motion and the impact effect are coupled.
\end{abstract}

Keywords-impact; dynamics; flexible multibody system; impulse momentum method (IMM)

\section{INTRODUCTION}

The accurate dynamic modeling method for flexible multibody systems is becoming more and more important in the engineering fields of aerospace, robotics, vehicles and advanced manufacturing. The dynamic equations of spatial flexible multi-link robot by using Lagrange method are derived [1-2]. Moreover, the impact dynamics of flexible multibody system has become one of the researching topics. The coupling of the flexible deformation and the large overall motion of flexible bodies needs to be considered, as well as the efficient and reasonable impact model. The proper handling of the impact process is the key to solve the impact dynamic problems of multibody system [3-4].

According to the different assumptions on the impact process, there are three types of impact models: the impulse momentum method (IMM), based on the generalized impulsemomentum equations and the coefficient of restitution equations; the continuous contact force method (CCFM), based on the quasi-static contact theory; and the contact constraint method (CCM), based on the kinematic constraint conditions of the impact process. There are a lot of impact models by far [510]. For IMM, the impact process is considered as an instantaneous process. The method is convenient for using since only the coefficient of restitution is needed. The solving efficiency is high because the impact time is not considered and no differential equations appear. Therefore, the impulse momentum model has been widely used in engineering.

The impact dynamics of chain flexible multibody system consisting of flexible links is to be studied in the paper. The influence of the coupling deformation items of the links on the system's dynamic behavior is considered. And the rigidflexible coupling continuous dynamic equations of flexible multibody system are established based on the recursive Lagrange dynamic modeling methods. Then the impact dynamic equations of the system are derived by using IMM, and the contact criterion is given. An impact dynamic simulation example is given, and the dynamic response is analyzed.

\section{DYNAMIC MODEL}

The flexible multibody system consists of $\mathrm{n}$ rigid joints and $\mathrm{n}$ flexible links, as shown in Figure 1. For link i, the length is $\mathrm{Li}$, the Young's modulus is $\mathrm{Ei}$, the shear modulus is $\mathrm{Gi}$, the moment of inertia on three direction is Ixi, Iyi, and Izi, the cross-sectional area is Axi, and the linear density is $\mu \mathrm{i}$. The flexible links are considered as slender Euler-Bernoulli beams composed with isotropic linear elastic materials, and are assumed to be with small deformation.

The multi-link flexible multibody system with large overall motion considers the links' tension, compression, bending and torsion deformation. In view of the limitations of traditional dynamic modeling methods, the coupling of large overall rigid body motion and flexible deformation motion is fully considered, and the rigid-flexible coupling dynamic model of the system is established.

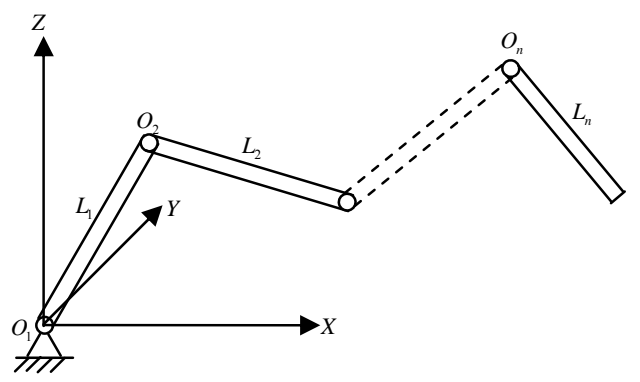

FIGURE I. FLEXIBLE MULTIBODY SYSTEM. 
On link $i$, the relative position array of a point $N(\eta, 0,0)$ after deformation in the form of homogeneous coordinates is shown in (1)- (2).

$$
\begin{aligned}
{ }^{i} \boldsymbol{h}_{i}(\eta, t) & =\left[\begin{array}{c}
1 \\
\eta \\
0 \\
0
\end{array}\right]+\sum_{j=1}^{N_{i}} \delta_{i j}(t)\left[\begin{array}{c}
0 \\
X_{i j}(\eta) \\
y_{i j}(\eta) \\
z_{i j}(\eta)
\end{array}\right]-\frac{1}{2} \sum_{j=1}^{N_{i}} \sum_{k=1}^{N_{i}} \delta_{i j}(t) \delta_{i k}(t)\left[\begin{array}{c}
0 \\
x_{i j k}(\eta) \\
0 \\
0
\end{array}\right] \\
\underline{X}_{i j k}(\eta) & =\int_{0}^{\eta}\left(\frac{\partial y_{i j}}{\partial \xi} \frac{\partial y_{i k}}{\partial \xi}+\frac{\partial z_{i j}}{\partial \xi} \frac{\partial z_{i k}}{\partial \xi}\right) \mathrm{d} \xi
\end{aligned}
$$

In which, $\operatorname{xij}(\eta), \operatorname{yij}(\eta)$ and $\operatorname{zij}(\eta)$ are the $j$-th order modal functions of link $i$ at position $\eta, \delta i j(t)$ is the $j$-th order modal coordinate of link $\mathrm{i}$. And $\mathrm{Ni}$ is the truncation number of modes for link i. In (1), the third item on the right side is the coupling deformation item by using the nonlinear strain field, which is the longitudinal shortening caused by transverse deformation, and $\underline{X}_{i j k}(\eta)$ is the coupling modal function. The traditional dynamic model ignores the coupling item, and the results could be divergent or instable when the large overall motion is on high speed

The absolute position vector hi of point $\mathrm{N}$ at the inertial coordinate system can be obtained by the homogeneous transformation matrices. Make Wi to be the homogeneous transform matrix from the inertial coordinate system $\mathrm{O}-\mathrm{XYZ}$ to the floating coordinate system of link $\mathrm{i}$, one can get that:

$$
\boldsymbol{h}_{i}=\boldsymbol{W}_{i} \cdot{ }^{i} \boldsymbol{h}_{i}
$$

Then the absolute velocity vector of point $\mathrm{N}$ can be obtained by making derivation of (3).

$$
\dot{\boldsymbol{h}}_{i}=\frac{\mathrm{d} \boldsymbol{h}_{i}}{\mathrm{~d} t}=\dot{\boldsymbol{W}}_{i} \cdot{ }^{i} \boldsymbol{h}_{i}+\boldsymbol{W}_{i} \cdot{ }^{i} \dot{\boldsymbol{h}}_{i}
$$

According to (3) and (4), the displacement and velocity of every point on each link can be obtained. And the entire system's motion can be achieved.

\section{CONTINUOUS DYNAMIC EQUATIONS}

The generalized coordinate for joint $\mathrm{i}$ is the angular displacement $\theta \mathrm{i}$, and the generalized coordinates for link $\mathrm{i}$ are the modal coordinates $\delta \mathrm{ij}(\mathrm{t}) \quad(1 \leq \mathrm{j} \leq \mathrm{Ni})$. Therefore, the generalized coordinates array of the multi-link flexible multibody system is selected as follows.

$$
\boldsymbol{q}=\left[\theta_{1}, \delta_{11}, \mathrm{~L}, \delta_{1 N_{1}}, \mathrm{~L}, \theta_{i}, \delta_{i 1}, \mathrm{~L}, \delta_{i N_{i}}, \mathrm{~L}, \theta_{n}, \delta_{n 1}, \mathrm{~L}, \delta_{n N_{n}}\right]^{\mathrm{T}}
$$

The kinetic energy $\mathrm{T}$ and the potential energy $\mathrm{V}$ of the system are calculated. Then the expressions of the kinetic energy and the potential energy are substituted into the Lagrange equations.

$$
\frac{\mathrm{d}}{\mathrm{d} t}\left(\frac{\partial T}{\partial \dot{\boldsymbol{q}}}\right)-\frac{\partial T}{\partial \boldsymbol{q}}+\frac{\partial V}{\partial \boldsymbol{q}}=\boldsymbol{F}_{\tau}
$$

Where $\mathrm{F} \tau$ is the generalized force corresponding to the nonconservative force. Then the rigid-flexible coupling continuous dynamic equations of the system can be obtained.

$$
\boldsymbol{M} \ddot{\boldsymbol{q}}=\boldsymbol{Q}
$$

In which, $\mathrm{M}$ is the generalized mass matrix of the system, and $\mathrm{Q}$ is the generalized force array of the system.

\section{IMPACT DYNAMIC EQUATIONS}

IMM is an impact solving method on the use of the generalized impulse momentum equations and the coefficient of restitution equations. The method comes from the classical impact theory of rigid bodies, and can be used for the impact dynamics of flexible bodies. The following assumptions are made: the impact time is infinitesimal, so the impact finishes instantaneously; the positions and orientations of all bodies do not change during impact; the shapes and inertia of all bodies do not change during impact.

In the impact process, the Lagrange equations are still tenable.

$$
\frac{\mathrm{d}}{\mathrm{d} t}\left(\frac{\partial T}{\partial \dot{\boldsymbol{q}}}\right)-\frac{\partial T}{\partial \boldsymbol{q}}+\frac{\partial V}{\partial \boldsymbol{q}}+\frac{\partial V_{I}}{\partial \boldsymbol{q}}=\boldsymbol{F}_{\tau}
$$

Where VI is the potential energy of the impact force.

In the infinitesimal impact time $\Delta t$, Equation (8) is integrated by time, and make $\Delta \mathrm{t} \rightarrow 0$. During $\Delta \mathrm{t}$, the positions and orientations of the system do not change, while the velocities mutate. Therefore, after integration and limit operation, the items in (8) are zero except those related with velocity and impact force.

$$
\Delta\left(\frac{\partial T}{\partial \dot{\boldsymbol{q}}}\right)+\frac{\partial \hat{V}_{I}}{\partial \boldsymbol{q}}=\mathbf{0}
$$

After deriving, one can get that:

$$
\boldsymbol{M} \Delta \dot{\boldsymbol{q}}=-\frac{\partial \hat{V}_{I}}{\partial \boldsymbol{q}}
$$


Where $\Delta \boldsymbol{\phi}_{\text {is }}$ the increment array of the generalized velocity, and $\hat{V}_{I}$ is the potential energy of the impact impulse which is similar with VI. A point $\mathrm{P}$ on link $\mathrm{k}$ of the flexible multibody system has normal impact with the external body.

$$
\hat{V}_{I}=-\boldsymbol{n}_{k}^{\mathrm{T}} I_{k}\left(\boldsymbol{W}_{k} \cdot{ }^{k} \boldsymbol{h}_{k}\right)
$$

In which, nk is the orientation vector of impact at $\mathrm{O}-\mathrm{XYZ}$, ${ }^{k} h_{k}$

is the position vector of the impacting point $\mathrm{P}$, and Ik is the impulse.

Combing (10) and (11), one can get the generalized impulse momentum equations. After adding in the coefficient of restitution equations, the IMM impact dynamic equations of flexible multibody systems can be established.

$$
\left\{\begin{array}{c}
\boldsymbol{M} \Delta \dot{\boldsymbol{q}}=\boldsymbol{D} I_{k} \\
\boldsymbol{n}_{k}^{\mathrm{T}} \cdot\left(\Delta \boldsymbol{V}_{k^{\mathrm{II}}}-\Delta \boldsymbol{V}_{k^{\mathrm{I}}}\right)=-(1+e) \boldsymbol{n}_{k}^{\mathrm{T}} \cdot\left(\boldsymbol{V}_{k^{\mathrm{II}}}-\boldsymbol{V}_{k^{\mathrm{I}}}\right)
\end{array}\right.
$$

Where e is the coefficient of restitution, $\boldsymbol{V}_{k^{\mathrm{I}}}$ and $\boldsymbol{V}_{k^{\mathrm{II}}}$ are the velocity array of the two impacting points at the impact moment, and $\mathrm{D}$ is an array.

By calculating the impact dynamic equations (12) in the form of algebraic equations, the impulse and the increment of the generalized velocity will be got, and then the state of motion of the system after impact can be obtained. After impact, the generalized coordinates remain unchanged, while the generalized velocities mutate.

$$
\left\{\begin{array}{c}
\left.\dot{\boldsymbol{q}}\right|_{t^{+}}=\left.\dot{\boldsymbol{q}}\right|_{t^{-}}+\Delta \dot{\boldsymbol{q}} \\
\left.\boldsymbol{q}\right|_{t^{+}}=\left.\boldsymbol{q}\right|_{t^{-}}
\end{array}\right.
$$

In the global dynamic solving process, the normal distance between the impacting points on the two impacting bodies is checked in real time. If the distance is less than 0 , the impact happens and the dynamic equations of the system are (12); otherwise the two impacting bodies do not contact with each other, and the dynamic equations of the system are (7).

\section{SiMULATION}

An impact simulation example of a two-link flexible multibody system is given, as shown in Figure 2.

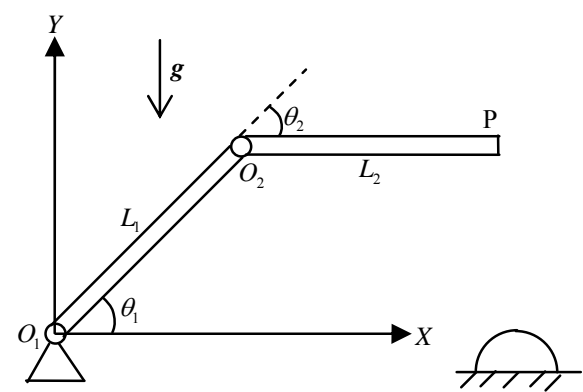

FIGURE II. TWO-LINK FLEXIBLE MULTIBODY SYSTEM.

The system falls freely from the initial angle is $\theta 1=\pi / 4$ and $\theta 2=-\pi / 4$, and the end point $\mathrm{P}$ of link 2 impact with the rigid $\mathrm{O}-$ $\mathrm{XZ}$ plane. The physical simulation time is $1.5 \mathrm{~s}$, the calculating time step is $5 \times 10-4 \mathrm{~s}$, and the coefficient of restitution e is 1 .

The time history of the angular displacement of the rotating joints is shown in Figure 3. The angular displacement of joint 1 decreases monotonically, yet the angular displacement of joint 2 decreases firstly and increases rapidly when the impact happens. The vertical displacement of the end point of the links is shown in Figure 4. The vertical displacement of link 1 drops continuously while the vertical displacement of link 2 decreases to 0 and then increases due to impact.

The change of the system's energy is shown in Figure 5. Before impact, the system's mechanical energy maintains conservation. In the impact process, the energy loss and transform is caused by impact. After impact, the system's mechanical energy maintains conservation again; however, the elastic potential energy is much larger than that before impact. The system's configuration is Figure 6, which intuitively reflects the changes of the system's position and orientation during the entire simulation process. Link 2 rebounds after impact, and link 1 still falls.

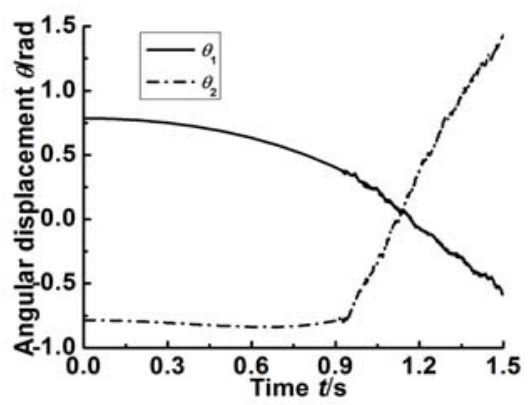

FIGURE III. ANGULAR DISPLACEMENT OF THE JOINTS. 


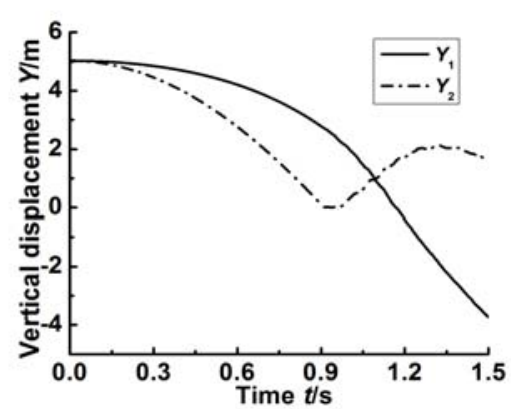

FIGURE IV. VERTICAL DISPLACEMENT OF THE LINKS.

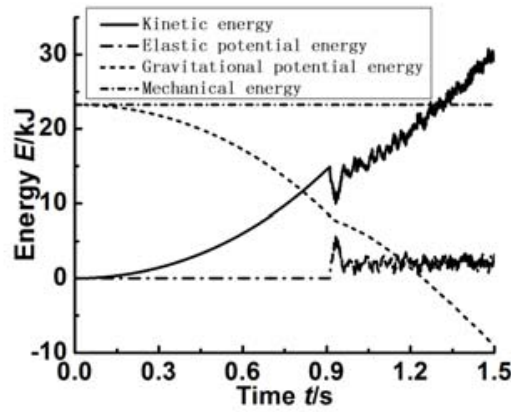

FIGURE V. SYSTEM'S ENERGY.

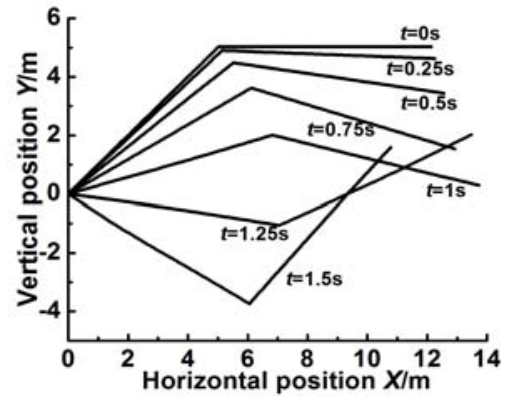

FIGURE VI. SYSTEM'S CONFIGURATION.

\section{CONCLUSIONS}

The rigid-flexible coupling impact dynamics of multi-link flexible multibody systems is studied. Firstly, based on the rigid-flexible coupling dynamic theory of flexible multibody system, the continuous dynamic equations of the system are established by using Lagrange method. Secondly, on the use of IMM, the impact dynamic equations are derived. The contact separation criterion is given, as well as the method of getting the dynamic behavior after impact. Thirdly, based on the impact dynamic theory of flexible multibody system, a simulation example for a two-link flexible multibody system is given, and the system's global dynamic response is shown. Studies show that the impact dynamic solving method can be used for global impact dynamics of flexible multibody systems. The dynamic behavior of the system changes obviously after impact. The credibility of the impact dynamic modeling method is verified by analyzing the simulation results. The large overall motion, the small deformation motion and impact effect are coupled.

\section{ACKNOWLEDGMENT}

This work was financially supported by the National Natural Science Foundation of China $(11402234,51107121)$ and the Key Scientific Research Foundation of Henan Province, China (16A130005).

\section{REFERENCES}

[1] Y. C. Duan, D. G. Zhang, and J. Z. Hong, "Partition method for impact dynamics of flexible multibody systems based on contact constraint", Applied Mathematics and Mechanics (English Edition), vol. 34, pp. 1393-1404, November 2013.

[2] C. L. You, J. Z. Hong, and G. P. Cai, "Modeling study of planar flexible manipulator undergoing large deformation," Journal of Shanghai Jiaotong University (Science), vol. E12, pp. 824-830, June 2007.

[3] Y. A. Khulief, "Modeling of impact in multibody systems: an overview," Journal of Computational and Nonlinear Dynamics, vol. 8, pp. 021012.1-021012.15, April 2013.

[4] W. Schiehlen, "Research trends in multibody system dynamics," Multibody System Dynamics, vol. 18, pp. 3-13, May 2007.

[5] S. K. Dwivedy and P. Eberhard, "Dynamic analysis of flexible manipulators, a literature review," Mechanism and Machine Theory, vol. 41, pp. 749-777, March 2006.

[6] D. M. Flickinger and A. Bowling, "Simultaneous oblique impacts and contacts in multibody systems with friction," Multibody System Dynamics, vol. 23, pp. 249-261, January 2010.

[7] Y. C. Duan, D. G. Zhang, and J. Z. Hong, "Global impact dynamic modeling and verification of a flexible beam with large overall motion," Advances in Mechanical Engineering, vol. 2013, pp. 1-8, November 2013.

[8] D. G. Zhang and J. Angeles, "Impact dynamics of flexible-joint robots," Computers and Structures, vol. 83, pp. 25-33, January 2005.

[9] M. Payr and C. Glocker, "Oblique frictional impact of a bar: analysis and comparison of different impact laws," Nonlinear Dynamics, vol. 41, pp. 361-383, January 2005.

[10] A. S. Yigit and A. P. Christoforou, "Impact dynamics of composite beams," Composite Structures, vol. 32, pp. 187-195, January 1995. 